\title{
ANALYSIS OF HYBRID CSA-DEA METHOD FOR FAULT DETECTION OF CRACKED STRUCTURES
}

\author{
SASMita SAHU \\ School of Mechanical Engineering, Kalinga Institute of Industrial Technology, KIIT University, Bhubaneswar, Odisha, \\ India; e-mail: gudusasmita@gmail.com
}

Priyadarshi Biplab Kumar, Dayal R. Parhi

Department of Mechanical Engineering, National Institute of Technology, Rourkela, Odisha, India

e-mail:p.biplabkumar@gmail.com; dayaldoc@yahoo.com

\begin{abstract}
Formation of damage in a structural element often causes failures which is not desirable at all by a maintenance team. Identification of location and severity of damage can aid in taking necessary steps to reduce catastrophic failures of structures. As a result, non-destructive methods of damage detection have gained popularity over the last few years. In this paper, a method of damage detection is proposed to identify the location and severity of damage by hybridising a clonal selection algorithm with a differential evolution algorithm. The inputs to the hybrid system are the relative values of the first three natural frequencies of the damaged structure, and the outputs are relative crack locations and relative crack depths. For training the hybrid system, the natural frequencies are found out using finite element analysis and experimental analysis for different crack locations and crack depths. The test results from the proposed hybrid method are compared with finite element analysis and experimental analysis for validation, and satisfactory outcomes have been observed.
\end{abstract}

Keywords: damage, CSA, DEA, hybridisation

\section{Introduction}

Dynamic characteristics are important factors which should be taken into consideration during designing of structures subjected to vibration. A change in dynamic properties of the structures shows changes in the system. This can be used for identification of faults in structural elements. So the fault detection of the structures has gained popularity for decades. Guo and Sun (2011) studied fault detection using a frequency response function. Sinha et al. (2002) predicted location of cracks in beam structures using measured vibration data. Caddemi and Morassi (2013) used mathematical modelling of damage using vibration data in multi-cracked Euler-Bernoulli beams. Most of the techniques developed for this problem were based on modal analysis of the vibrating structures. Pawar and Sawant (2014) identified the effect of non-linearities, namely, material, geometric and damping on natural frequencies and mode shapes of a cracked cantilever beam by theoretical, numerical and experimental methods. Rahimi and Soltani (2015) proposed a comprehensive closed-form approach to determine the dynamic response of single degree-of-freedom and multi degree-of-freedom systems subjected to stationary and non-stationary Gaussian excitations. Pölöskei and Szekrényes $(2017,2018)$ discussed using a higher order theory for dynamic stability analysis and a quasi-periodic excitation in a structurally damped delaminated composite beam. Manoach et al. (2016, 2017) discussed vibration based damage detection methods and dynamics of composite beams.

There have been considerable attempts to understand and model the dynamics of a vibrating cracked beam. All models arising from theoretical consideration have a large number of assumptions to be made. Therefore, there is a need to use other approaches such as Artificial Intelligence 
(AI), techniques to find the severity of damage in a cracked structure. Xu et al. (2015) proposed an efficient and powerful Bayesian Network for identification of faults in an uncertain dynamic environment. Ling et al. (2009) discussed regarding a modified clonal selection algorithm in an engineering application. Clonal selection algorithm (CSA) is a type of Artificial Immune System (AIS) based on the clonal selection principle (CSP). De Castro and von Zuben (2000) investigated the potential of the CSA method to be used as an engineering problem solving method. Xie et al. (2013) presented the theory of CSP that recounts the immune response when the antigens are identified. Despite of several advantages in good approximation for finding the global solution in different multimodal problems, the major shortcoming of CSA is identified as premature and slow convergence. Owing to prematurity, the CSA method usually fails to search for new possible solutions. For this reason, the method generally gets trapped in one of local solutions. The CSA method is used to converge slowly when searching for better solutions in high dimensional problems. Recently, many investigations have been made to control these limitations; some of the researchers have used elimination of the oldest candidate solutions and employment of the chaos-based mutation strategy for improving the diversity of the possible candidate solutions. Campelo et al. (2005) and Timmis et al. (2008) proposed a local search technique and immune memory encoding by incorporating into the standard CSA method to enhance exploitation of the population. Alternatively, other algorithms which can be integrated in the standard CSA method are incorporated. The differential evolution algorithms (DEA) have also been used to improve the searching capability of the CSA method. Qin and Suganthan (2005) used a self-adaptive DE for numerical optimisation. Tang (2012) used evolutionary operations such as the DEA for parameter estimation of the Wiener model. Abdullah et al. (2011) presented a method to reproduce and relocate antibodies to enhance fitness values by integrating CSA with an evolutionary algorithm. Gong (2012) and Gong et al. (2012) discussed a high performance immune system in their work. Garain et al. (2006) used CSA for recognition of handwritten Indic script. Brest and Maučec (2011) proposed a self-adaptive DEA using population size reduction and three strategies which can also be used in different fields of engineering.

In this research work, evolutionary operations of the DE method are used for improving the exploration capability of the standard CSA. The proposed method has been designed by hybridising CSA and DEA techniques. In this hybrid method (CSA-DEA), the crossover and mutation operations are applied to exploit the information regarding different antibodies in the population. This method can efficiently improve the searching quality compared to the standard CSA and DEA methods. The architecture for applying the hybrid method has been given in Fig. 1. This methodology has been adopted for finding out the relative crack depth and crack positions from the relative natural frequencies of the vibrating system.

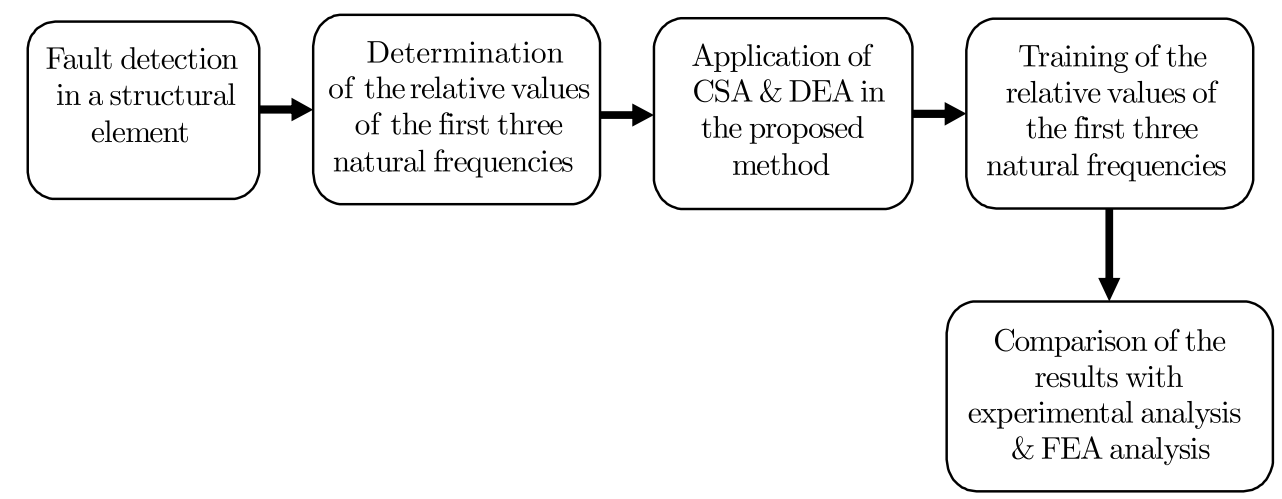

Fig. 1. Block diagram to find severity of damage in a cracked structure

Here, the first three natural frequencies are extracted from FEA and converted into relative values. The relative values of the natural frequencies $(r f n f, r s n f, r t n f)$ are found out by 
comparing the natural frequencies of the uncracked and cracked beam. Relative values of the crack depth and crack location $(r c d, r c l)$ are also found out using a similar method. This work considers only the natural frequencies because they are less prone to error while calculating. The relative first three natural frequencies are treated as the input variables for the proposed method. The outputs from the system are the relative values of the crack depth and crack location which, in turn, contain the information of damage severity. The proposed method is a type of an inverse analysis problem. Here, by giving the crack depths and crack locations, the natural frequencies are found using theoretical, finite element and experimental analyses. These sets of crack locations and first three natural frequencies are used as the dataset.

\section{Theoretical analysis on dynamics of cracked beam}

A fixed-fixed beam with a transverse hairline crack has been modeled in the present work.

Due to the presence of the crack, an additional flexibility is introduced which can be defined in matrix form. In this work, a $2 \times 2$ matrix is considered. The dimension of the matrix depends on the degrees of freedom. The transverse surface crack is having depth $a$, width $B$ and height $W$. Here, the beam is subjected to axial force $P_{1}$ and bending moment $P_{2}$ as shown in Fig. 2.

(a)

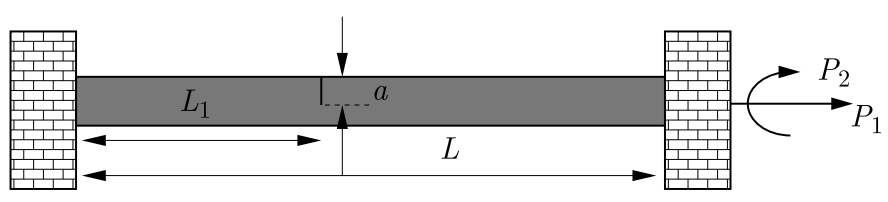

(b)

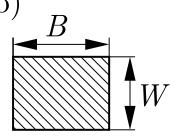

Fig. 2. (a) Cracked fixed-fixed beam, (b) cross sectional view of the cracked fixed-fixed beam

Due to the formation of the crack, some energy is released at the cracked section. This is known as strain energy, and it is a type of potential energy. As per Tada et al. (1973), the strain energy release rate (SR) at the cracked section is given below

$$
S_{R}=\frac{1}{E^{\prime}}\left(K_{11}+K_{12}\right)^{2}
$$

where

$$
\frac{1}{E^{\prime}}= \begin{cases}\frac{1-\nu^{2}}{E} & \text { for plain strain condition } \\ \frac{1}{E} & \text { for plain stress condition }\end{cases}
$$

where $\nu$ is Poisson's ratio, $K_{11}, K_{12}$ are the stress intensity factors of mode I (opening of the crack) for load $P_{1}$ and $P_{2}$, respectively. The values of the stress intensity factors can be written as (Tada et al., 1973)

$$
K_{11}=\frac{P_{1}}{B W} \sqrt{\pi a} F_{1}\left(\frac{a}{W}\right) \quad K_{12}=\frac{6 P_{2}}{B W^{2}} \sqrt{\pi a} F_{2}\left(\frac{a}{W}\right)
$$

where expressions for $F_{1}$ and $F_{2}$ are as follows

$$
\begin{aligned}
& F_{1}\left(\frac{a}{W}\right)=\sqrt{\frac{2 W}{\pi a} \tan \frac{\pi a}{2 W}}\left(\frac{0.752+2.02 \frac{a}{W}+0.37\left(1-\sin \frac{\pi a}{2 W}\right)^{3}}{\cos \frac{\pi a}{2 W}}\right) \\
& F_{2}\left(\frac{a}{W}\right)=\sqrt{\frac{2 W}{\pi a} \tan \frac{\pi a}{2 W}}\left(\frac{0.923+0.199\left(1-\sin \frac{\pi a}{2 W}\right)^{4}}{\cos \frac{\pi a}{2 W}}\right)
\end{aligned}
$$


Let $S_{E}$ be the strain energy due to the crack. Applying Castigliano's theorem, the additional displacement $u_{i}$ along the force $P_{i}$ can be derived as

$$
u_{i}=\frac{\partial S_{E}}{\partial P_{i}}
$$

The strain energy will have the form

$$
S_{E}=\int_{0}^{a} \frac{\partial S_{E}}{\partial a} d a=\int_{0}^{a} S_{R} d a
$$

where $S_{R}=\partial S_{E} / \partial a$ is the strain energy density function.

From (2.1) and (2.3), the additional displacement can be defined as

$$
u_{i}=\frac{\partial}{\partial P_{i}}\left(\int_{0}^{a} S_{R}(a) d a\right)
$$

The flexibility influence coefficient $C_{i j}$ can be written as

$$
C_{i j}=\frac{\partial u_{i}}{\partial P_{j}}=\frac{\partial^{2}}{\partial P_{i} \partial P_{j}} \int_{0}^{a} S_{R}(a) d a
$$

The final flexibility matrix can be found out as

$$
C_{i j}=\frac{\partial u_{i}}{\partial P_{j}}=\frac{\partial^{2}}{\partial P_{i} \partial P_{j}} \int_{-B / 2}^{+B / 2} \int_{0}^{a} S_{R}(a) d a d z
$$

Putting the value of the strain energy release rate, equation (2.9) is modified as

$$
C_{i j}=\frac{B}{E^{\prime}} \frac{\partial^{2}}{\partial P_{i} \partial P_{j}} \int_{0}^{a}\left(K_{11}+K_{12}\right)^{2} d a
$$

Putting $\xi=a / W, d \xi=d a / W, d a=W d \xi$, equation (2.10) is converted into

$$
C_{i j}=\frac{B W}{E^{\prime}} \frac{\partial^{2}}{\partial P_{i} \partial P_{j}} \int_{0}^{\xi}\left(K_{11}+K_{12}\right)^{2} d \xi
$$

and

$$
\begin{aligned}
& C_{11}=\frac{B W}{E^{\prime}} \int_{0}^{\xi} \frac{\pi a}{B^{2} W^{2}}\left(F_{1}(\xi)\right)^{2} d \xi=\frac{2 \pi}{E^{\prime}} \int_{0}^{\xi} \xi\left(F_{1}(\xi)\right)^{2} d \xi \\
& C_{12}=C_{21}=\frac{12 \pi}{E^{\prime} B W} \int_{0}^{\xi} \xi F_{1}(\xi) F_{2}(\xi) d \xi \quad C_{22}=\frac{72 \pi}{E^{\prime} B W} \int_{0}^{\xi} \xi F_{1}(\xi) F_{2}(\xi) d \xi
\end{aligned}
$$

Converting the influence co-efficient into dimensionless form

$$
\overline{C_{11}}=C_{11} \frac{E^{\prime} B}{2 \pi} \quad \overline{C_{12}}=C_{12} \frac{E^{\prime} B W}{12 \pi}=\overline{C_{21}} \quad \overline{C_{22}}=C_{22} \frac{E^{\prime} B W^{2}}{72 \pi}
$$

The local stiffness matrix can be obtained by taking the inversion of the compliance matrix. The nodal stiffness matrix is based on the compliance matrix calculation which is expressed by

$$
\mathbf{S}=\left[\begin{array}{ll}
S_{11} & S_{12} \\
S_{21} & S_{22}
\end{array}\right]=\left[\begin{array}{ll}
C_{11} & C_{12} \\
C_{21} & C_{22}
\end{array}\right]^{-1}
$$




\subsection{Analysis of vibration parameters of the beam element with a transverse crack}

Figure 3 represents $u_{1}(x, t), u_{2}(x, t)$ as the amplitudes of longitudinal vibration and $y_{1}(x, t)$, $y_{2}(x, t)$ as the amplitudes of bending vibration for the beam element before and after the crack. Here, the crack is assumed to be open during vibration, and the system is considered to be linear.

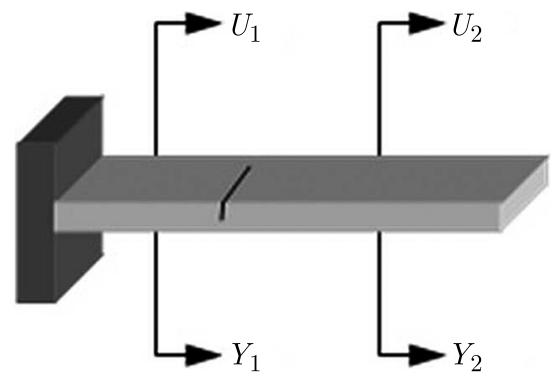

Fig. 3. Amplitudes of longitudinal and bending vibration

The normal functions for the system can be defined as

$$
\begin{aligned}
& \overline{U_{1}}(\bar{x})=A_{1} \cos \left(\overline{K_{u}} \bar{x}\right)+A_{2} \sin \left(\overline{K_{u}} \bar{x}\right) \quad \overline{U_{2}}(\bar{x})=A_{3} \cos \left(\overline{K_{u}} \bar{x}\right)+A_{4} \sin \left(\overline{K_{u}} \bar{x}\right) \\
& \overline{Y_{1}}(\bar{x})=A_{5} \cosh \left(\overline{K_{y}} \bar{x}\right)+A_{6} \sinh \left(\overline{K_{y}} \bar{x}\right)+A_{7} \cos \left(\overline{K_{y}} \bar{x}\right)+A_{8} \sin \left(\overline{K_{y}} \bar{x}\right) \\
& \overline{Y_{2}}(\bar{x})=A_{9} \cosh \left(\overline{K_{y}} \bar{x}\right)+A_{10} \sinh \left(\overline{K_{y}} \bar{x}\right)+A_{11} \cos \left(\overline{K_{y}} \bar{x}\right)+A_{12} \sin \left(\overline{K_{y}} \bar{x}\right)
\end{aligned}
$$

where

$$
\begin{array}{lcrrr}
\bar{x}=\frac{x}{L} & \bar{u}=\frac{u}{L} \quad \bar{y}=\frac{y}{L} & \beta=\frac{L_{1}}{L} & \overline{K_{u}}=\frac{\omega L}{C_{u}} \\
C_{u}=\sqrt{\frac{E}{\rho}} & \overline{K_{u}}=\sqrt{\frac{\omega L^{2}}{C_{y}}} & C_{y}=\sqrt{\frac{E I}{\mu}} & \mu=A \rho
\end{array}
$$

where $A_{i}(i=1, \ldots, 12)$ are constants which can be determined from boundary conditions of the beam. The boundary conditions of the fixed-fixed beam in consideration are

$$
\begin{array}{lll}
\overline{u_{1}}(0)=0 & \overline{Y_{1}}(0)=0 & {\overline{Y_{1}}}^{\prime}(0)=0 \\
{\overline{u_{2}}}^{\prime}(0)=0 & \overline{Y_{2}^{\prime \prime}}(1)=0 & \overline{Y_{2}}{ }^{\prime \prime \prime}(1)=0 \\
\overline{u_{1}}(\beta)=\overline{u_{2}}(\beta) & \overline{Y_{1}}(\beta)=\overline{Y_{2}}(\beta) & \\
\overline{Y_{1}}(\beta)=\overline{Y_{2}^{\prime \prime}}(\beta) & {\overline{Y_{1}}}^{\prime \prime \prime}(\beta)={\overline{Y_{2}}}^{\prime \prime \prime}(\beta) &
\end{array}
$$

The normal functions, along with the boundary conditions as mentioned above, yield the characteristic equation of the system as

$$
\operatorname{det} \mathbf{Q}=0
$$

This determinant is a function of the angular velocity $\omega$ and the local stiffness matrix which depends on the crack depth and crack location. The roots of equation (2.18) versus the angular velocity $\omega$ provide the eigenvalues (natural frequencies) of the system.

\section{Finite element analysis of cracked beam}

For vibration analysis of the uncracked and cracked cantilever beam, the ALGOR V 19.3 SP 2 Finite Element program is used. First, the beam element with different single cracks is plotted 
using CATIA V5R15 software, and then they are treated in the ALGOR environment. The uncracked and cracked beam model is then analysed in the ALGOR environment. First of all, mesh generation is performed. The mesh size is around $1.4529 \mathrm{~mm}$, and 33369 elements are created. Then the parameters such as the element type (brick and isotropic), material name (Aluminium Alloy) are defined in the ALGOR environment. After that, boundary conditions are given by constraining all degrees of freedom of the nodes located at the left end of the beam. The model unit is then changed to S.I. standards. Then in the analysis window, the particular analysis type is selected (natural frequency, i.e. modal analysis). Then the analysis is performed, and the first three modes of natural frequencies at different crack locations and crack depths of the fixed-fixed beam are recorded. Figure 4 shows modes of vibration of the cracked beam after finite element analysis.

(a)

(b)

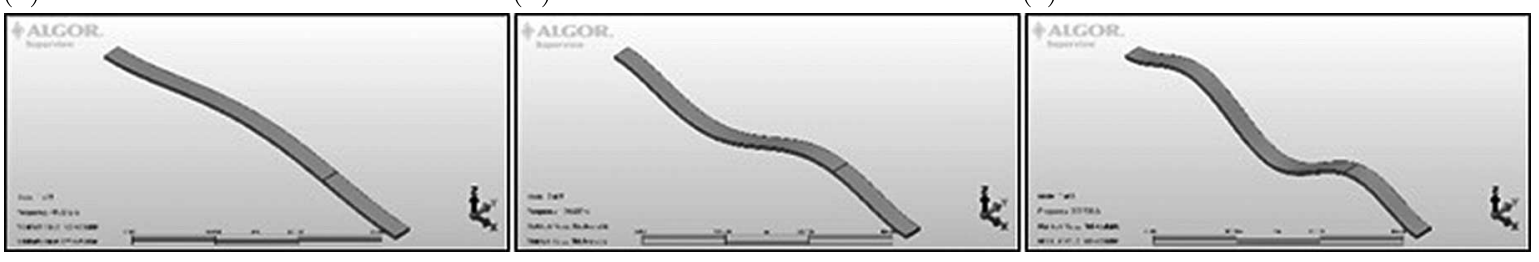

Fig. 4. First three modes of vibration of the cracked fixed-fixed beam

The length and cross-sectional area of the beam are $800 \mathrm{~mm}$ and $38 \times 8 \mathrm{~mm}^{2}$, respectively. As per material properties, the modulus of elasticity $E$ is $70000 \mathrm{MPa}$, density $\rho$ is $2700 \mathrm{~kg} / \mathrm{m}^{3}$.

\section{Analysis of fault detection using a simple clonal selection algorithm}

The clonal selection theory (CST) is a type of the AIS approach. The idea behind the CST is cloning of cells which are capable of identifying a foreign substance.

The CSA tells that when $B$ cells antigen receptors (antibodies) bind with an antigen, $B$ cells become activated and proliferate into clones. As the clones are not very improved, they are subjected to somatic hypermutation. CSA is the mechanism which guarantees diversity through this operation. The cloning rate is proportional to the fitness of the $B$ cells. The fittest cell differentiates into clones the most. Then the mutated cells form plasma cells and memory cells. The memory cells $M$ commonly continue in the host for promoting a fast secondary response in subsequent encounters with the same antigen. Figure 5 summarises the CST.

The antibodies $(\mathrm{Ab})$ in the current investigation are consisting of rfnf, rsnf, rtnf, rcd and $r c l$. The antigens are chosen randomly (Ag i.e. rfnf, rsnf and $r t n f)$ and are presented to the antibodies in the collection or stock (dataset). The antigens represent the data from the field, i.e., the values given from the working area. Here, each antibody and antigen is coded using a binary encoding scheme. Then the antibody population is subjected to affinity measurement. In the present work, the hamming distance method is used for the affinity matching operation. The antibodies and antigens are presented in Fig. 6 .

The clonal selection algorithm is described as follows:

1. First, the antibodies and antigens are initialized and encoded. Each antibody represents a solution containing the parameters (rfnf, rsnf, rtnf, $r c d$ and $r c l$ ) of the given problem.

2. All the antibodies (solutions) in the initial population are trained in the fitness function (affinity function) with respect to the antigens ( $r f n f$, rsnf and $r$ tnf $)$. 


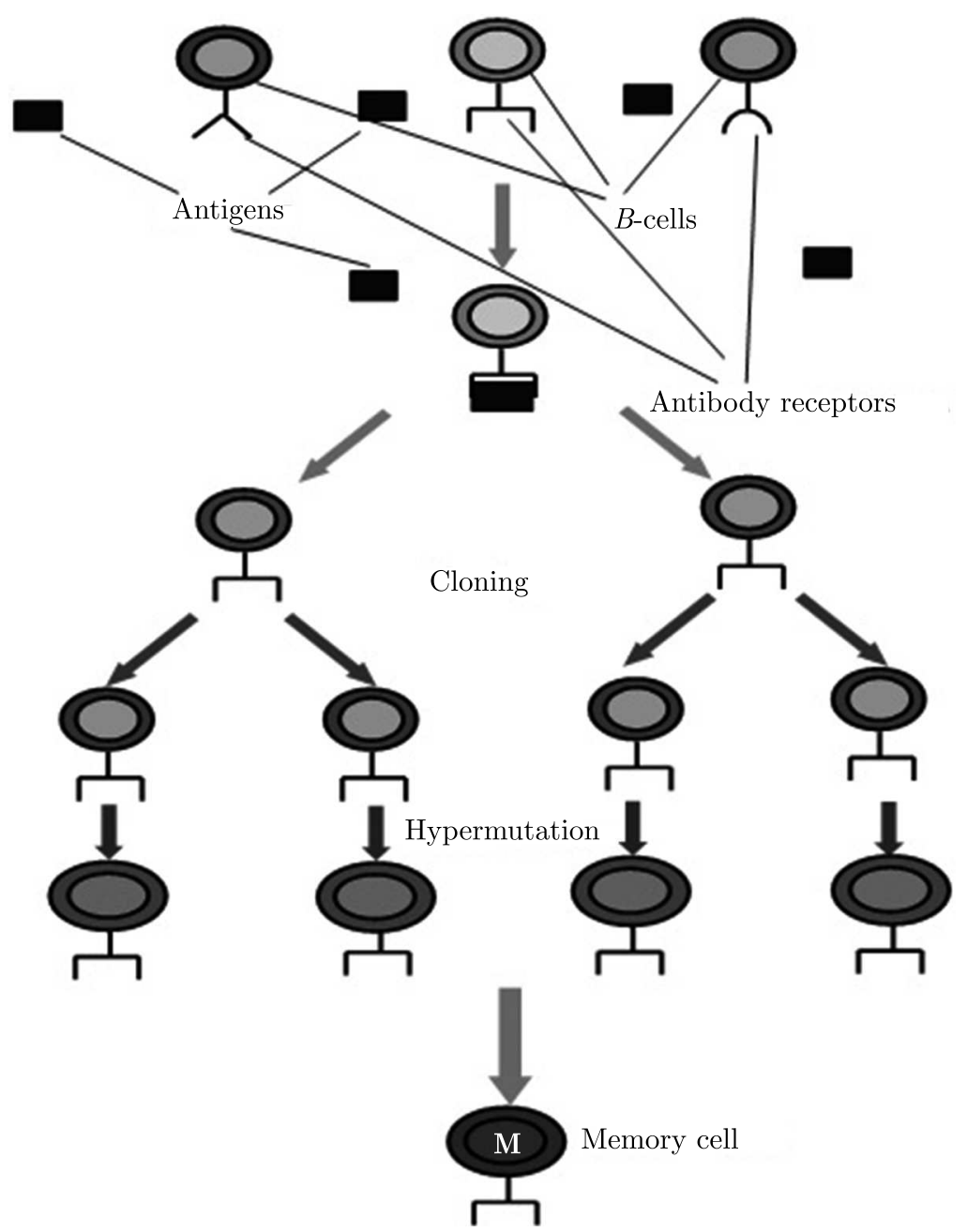

Fig. 5. Pictorial representation of the clonal selection algorithm

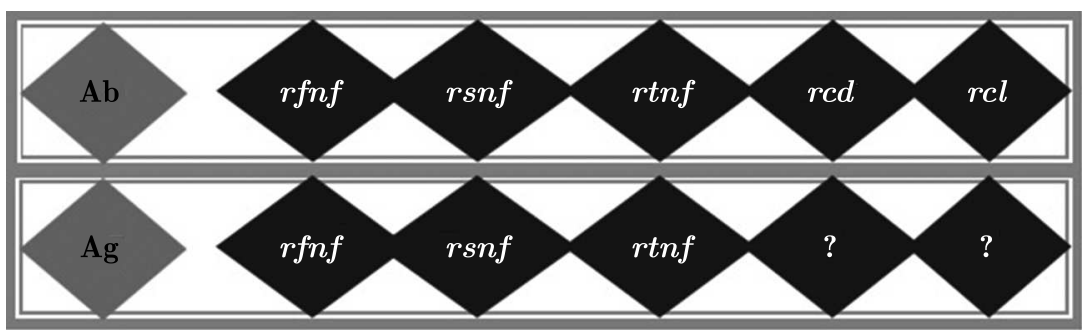

Fig. 6. Presentation of the antibodies and antigens

3. The antibodies are selected according to their fitness rank w.r.t antigens

Fitness function

$$
=\sqrt{\left(r f n f_{f l d}-r f n f_{x_{1, i}}\right)^{2}+\left(r s n f_{f l d}-r s n f_{x_{1, i}}\right)^{2}+\left(r t n f_{f l d}-r t n f_{x_{1, i}}\right)^{2}}
$$

$r f n f_{f l d}, r s n f_{f l d}, r t n f_{f l d}=$ first three relative natural frequencies of the field.

4. For each selected antibody, clones are generated. The rate of cloning is directly proportional to the affinity measurement. Clones of the selected antibodies are produced according to the affinity proportion.

5. These cloned antibodies are then mutated using the hypermutaion operation of CSA. The rate of mutation is indirectly proportional to the affinity measurement. 
6. The affinity measurement function is again applied to the mutated population of clones, and the highest ranking antibody is selected as the candidate memory cell. If its affinity score is more than that of the current memory cell, then it becomes a new memory cell.

7. Antibodies with a lower fitness from the population are removed and replaced by newly generated higher ranking antibodies to form a new population for the next iteration.

8. Steps from 2-7 are repeated in each iteration till the algorithm meets the threshold values.

Table 2 provides the results of CSA.

\section{Analysis of fault detection using simple differential evolution algorithm}

Differential evolution which utilizes $N_{p} D$-dimensional vectors of real valued parameters is a parallel direct search method. $P^{(G)}$ is the current population composed of encoded individuals $\mathbf{X}_{i}$. Figure 7 describes the idea of DEA which has been used for crack detection in the current research work.

Here $\mathbf{X}_{i}=\left[x_{1}, x_{2}, x_{3}, x_{4}, x_{5}\right]$, where $x_{1}=r f n f, x_{2}=r s n f, x_{3}=r t n f, x_{4}=r c d, x_{5}=r c l$, $G$ - number of generations, $D$ - number of parameters.

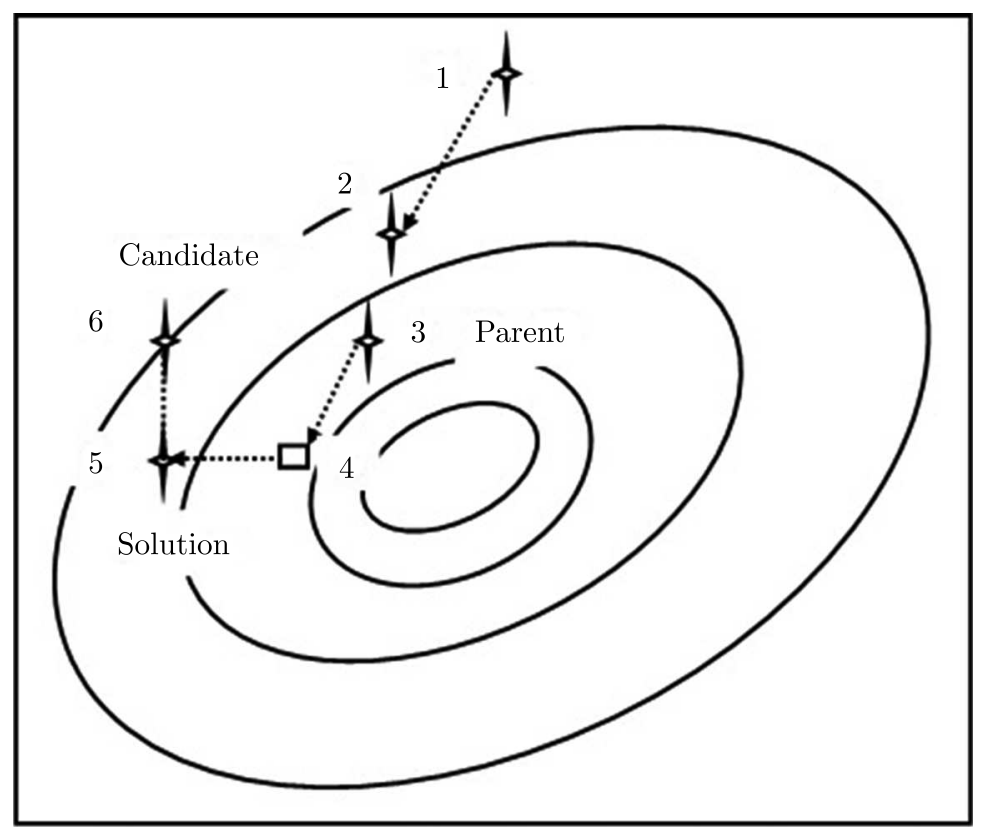

Fig. 7. Differential evolution algorithm

The following steps describe the working of DEA.

1. Read the parameters - scaling factor $F$, crossover constant $C_{r}$, population size $N_{p}$, maximum iterations $G_{\max }$ and decision variables $D$ (i.e. $r f n f$, rsnf, rtnf, $r c d$ and $r c l$ ).

2. Set the iteration $G=0$, population index $i=1$, decision variable $j=1$.

3. Initialise the parent vector uniformly in the random search space. The initial value of the $j$-th parameter in the $i$-th individual at the generation $G=0$ is given as

$$
x_{j, i}^{(0)}=x_{j}^{\min }+\operatorname{rand}_{j}(0,1)\left(x_{j}^{\max }-x_{j}^{\min } \quad j=1, \ldots, D(5) \quad i=1, \ldots, N_{p}\right.
$$

4. Once initialised, DE mutates the population to produce a population of $N_{p}$ mutant vectors

$$
\mathbf{X}_{i}^{\prime(G)}=\mathbf{X}_{a}^{(G)}+F\left(\mathbf{X}_{b}^{(G)}-\mathbf{X}_{c}^{(G)}\right) \quad i=1, \ldots, N_{p}
$$


The indices $a, b$ and $c$ are randomly chosen. These indices are different from each other and form the base vector index $i$. The scaling factor $F$ is a real and constant factor $F \in[0,2]$.

5. After mutation, uniform crossover is employed to generate trial vectors $\mathbf{X}_{i}^{\prime \prime}$ by mixing the mutant vectors and target vectors $\mathbf{X}_{i}$

$$
X_{j, i}^{\prime \prime}(G)= \begin{cases}X_{j, i}^{\prime(G)} & \text { if } \operatorname{rand}_{j}(0,1) \leqslant C_{r} \quad \text { or } \quad j=j_{\text {rand }} \\ X_{j, i}^{(G)} & \text { otherwise }\end{cases}
$$

The crossover operation is applied to each pair of the target vector $\mathbf{X}_{i}$ and its corresponding mutant vector $\mathbf{X}_{i}^{\prime}$ to generate a trial vector $\mathbf{X}_{i}^{\prime \prime}$.

The crossover probability $C_{r} \in(0,1)$ is a user defined value. The crossover operator copies the $j$-th parameter of the mutant vector $\mathbf{X}_{i}^{\prime}$ to the corresponding parameter of the trial vector $\mathbf{X}_{i}^{\prime \prime}$, if $\operatorname{rand}_{j}(0,1) \leqslant C_{r}$, otherwise it is copied from the corresponding target vector $\mathbf{X}_{i}$.

6. Sometimes, the upper and lower bounds of the newly generated trial vectors exceed the given value, and then they are randomly and uniformly initialised to the initial value given previously. The objective function values of all trial vectors are evaluated. Then the selection operator (according to the fitness rank) determines the population by choosing between the trial vectors and their predecessors (target vectors):

a) if the trial vector $\mathbf{X}_{i}^{\prime \prime}(G)$ has an equal or lower fitness function value (optimal) than that of its target vector $\mathbf{X}_{i}^{(G)}$, it replaces the target vector in the next generation.

b) otherwise, the target vector retains its place in the population for at least one more generation

$$
\mathbf{X}_{i}^{(G+1)}= \begin{cases}\mathbf{X}_{j, i}^{\prime(G)} & \text { if } f\left(\mathbf{X}_{i}^{\prime \prime}(G)\right) \leqslant f\left(\mathbf{X}_{i}^{(G)}\right) \\ \mathbf{X}_{i}^{(G)} & \text { otherwise }\end{cases}
$$

Once the new population is installed, the process of mutation, crossover and selection are repeated for several generations.

7. The algorithm stops after reaching threshold values for the target vector.

Table 2 gives the results of the differential evolution algorithm.

\section{Analysis of fault detection using fusion of evolutionary CSA-DEA}

Like other hybridized methods, these two nature inspired algorithms are incorporated to overcome one another's limitations. As they are integrated together, they retain some part of their original algorithms features. Like the general CSA, the population of antibodies in the hybridized system (CSA-DEA) is initialised randomly. Then similarity measurement is done to find the affinity of the antibodies for the antigens. There is no change in the antibody and antigen definition and representation. Then on the basis of the affinity measurement, some of the antibodies are selected for the cloning process. The mutation and crossover are done using DEA on the clones, and a new generation of antibodies is produced. After mutation and crossover, a selection operator of DEA is applied on the original and offspring antibodies. The antibodies with poor affinity values undergo randomisation according to DEA. These selected improved antibodies form a new population. The antibody which produces the best affinity value is chosen as the current best antibody, and the algorithm is repeated till it meets the stopping criteria.

In this hybrid scheme, DEA is used after initialisation and encoding of the antibodies, mutation and crossover operation of the simple CSA. By employment of the evolutionary operations of the DEA method in the mutation and crossover operation of the standalone CSA, the fitness 
values of different clones are enhanced. In the modified version of CSA, by the use of mutation and crossover operations from DEA, the information of neighbouring clones is included against the standard mutation operation which considers only the single clone and its original antibody in the standard CSA method.

Following are the steps used in the hybridisation of CSA and DEA:

1. The initial population and fitness function are defined. Antibodies and antigens are encoded.

2. Individuals in the initial population are evaluated using affinity measurement and ranked according to their fitness values.

3. Individuals $(n)$ according to their fitness values are selected.

4. The antibodies according to their affinity values undergo proliferation to create clones $C$. The number of clones is directly proportional to the affinity values or fitness values. Figure 8 describes the cloning process in the CSA-DEA hybridized method.

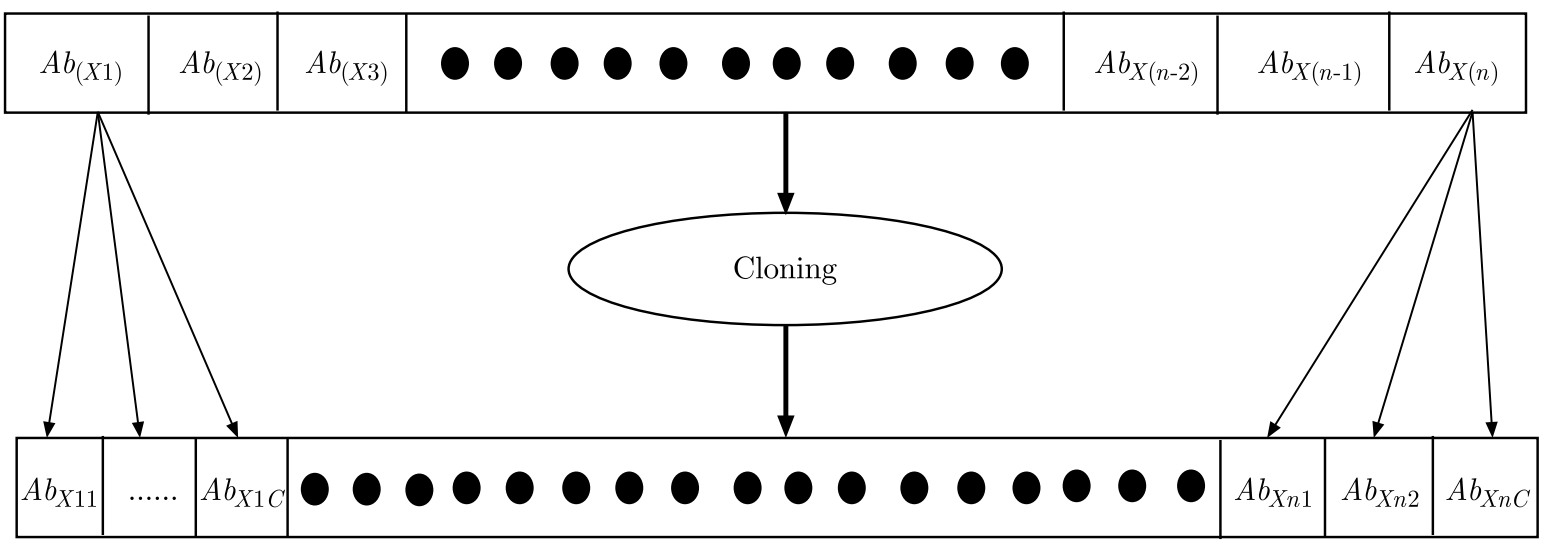

Fig. 8. Cloning process applied to DEA individuals in CSA-DEA

5. The clones generated by proliferation undergo mutation using equation (5.2) of DEA.

6. The mutated clones undergo crossover using equation (5.3) of DEA.

7. After crossover, the selection operation is applied on all the antibodies using equation (5.4) of DEA.

8. Then antibodies with poor affinity values are randomized using equation (5.1) of DEA.

9. These antibodies create a new population in the next generation.

10. If the stopping criteria are met then the antibody with the highest affinity is chosen as the current best antibody, otherwise the algorithm is repeated. The stopping criteria for the algorithms are i) treatment of all the antigens to the antibody population, ii) number of iterations (50), and iii) time elapse (3 minutes). The algorithm terminates if it faces any one of the stopping conditions first.

Figure 9 describes the flow chart for the CSA-DEA hybridisation technique.

\section{Experimental set-up used in fault detection of the cracked beam}

The instruments used in the free vibration analysis of the fixed-fixed beam are an impact hammer, vibration pick-up, vibration analyser and vibration indicator (Fig. 10). Using the impact hammer, the cracked fixed-fixed beam is excited in the free vibration mode. The vibration analyser is PULSE LAB Prolite 3560. The excitation parameters are picked up by the vibration pick-up or accelerometer. Then these parameters are fed to the vibration analyser where the parameters are analysed, and the results are shown in the vibration indicator. 


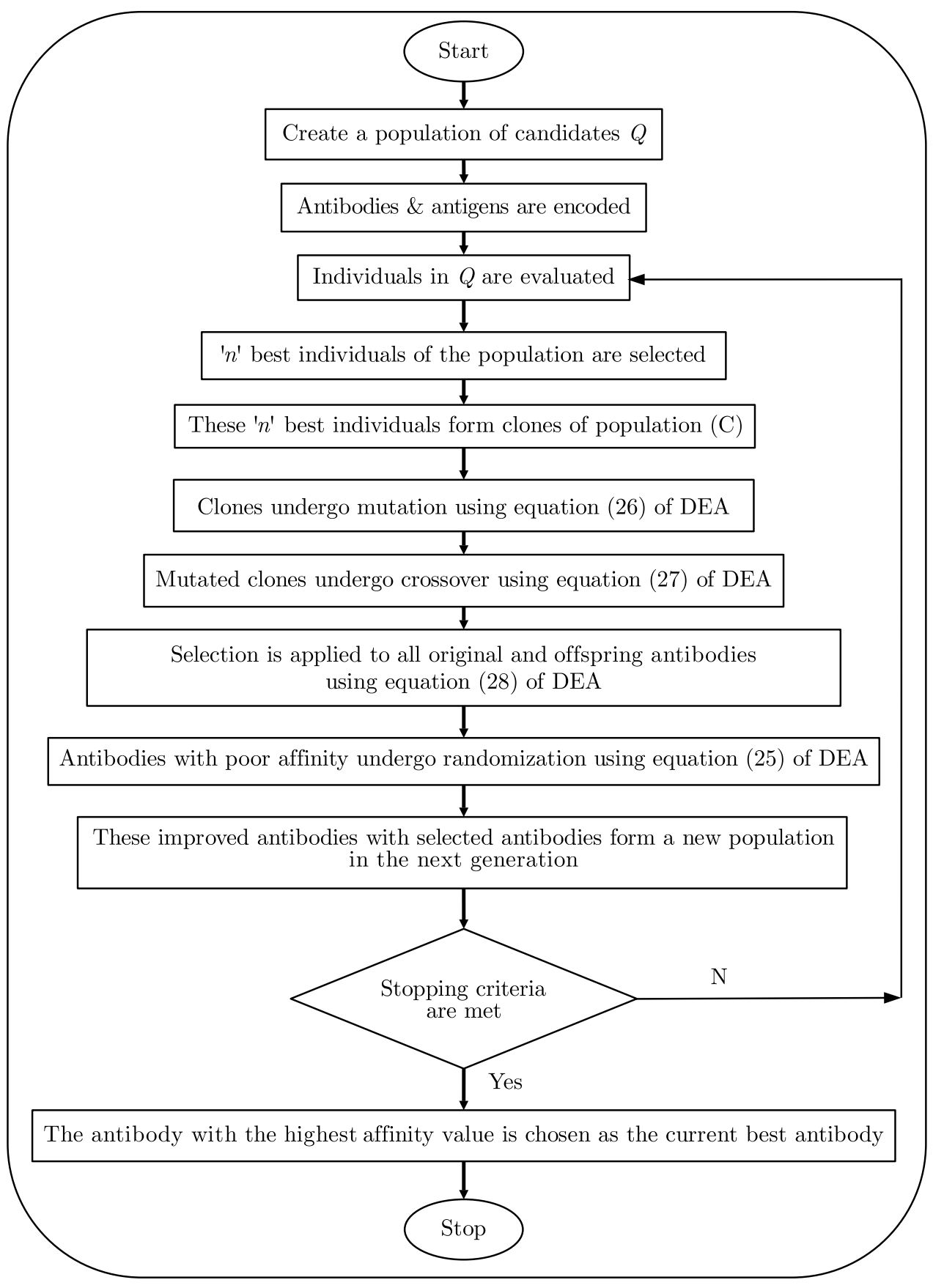

Fig. 9. Flow chart of the CSA-DEA algorithm

Several tests are conducted using the experimental setup on aluminium alloy beam specimens $(800 \mathrm{~mm} \times 38 \mathrm{~mm} \times 6 \mathrm{~mm})$ with a transverse crack for determining the natural frequencies at different crack locations and crack depths. These specimens are given vibration by the impact hammer, and the 1st, 2nd and 3rd natural frequencies are recorded in the vibration indicator.

\section{Results and discussion}

The following Tables 1-3 show the results obtained from the application of the proposed technique. 


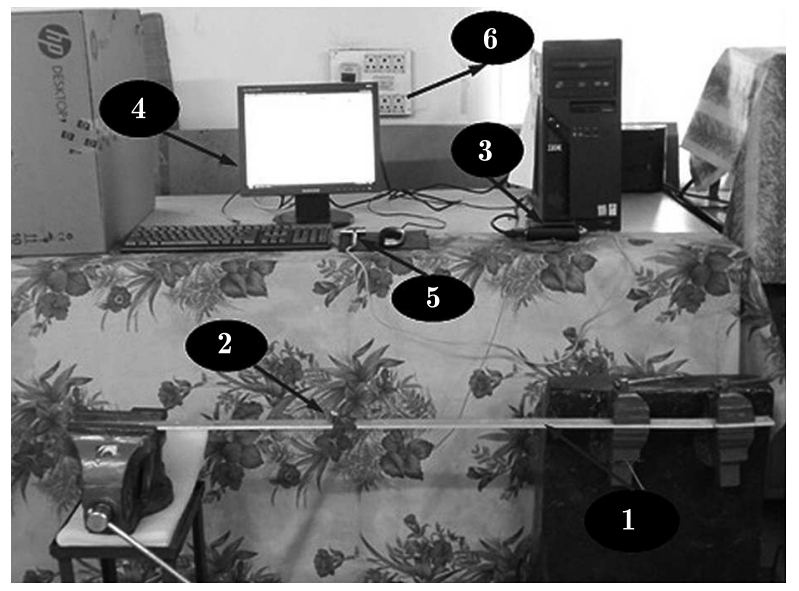

Fig. 10. Experimental set-up; 1 - cracked fixed-fixed beam with a single crack, 2 - vibration pick-up, 3 - vibration analyser, 4 - vibration indicator, 5 - impact hammer, 6 - power distribution

Table 1. Results of hybridisation of CSA and DEA

\begin{tabular}{|c|c|c|c|c|c|c|c|c|c|}
\hline \multirow{2}{*}{ No. } & \multicolumn{5}{|c|}{ FEA results } & \multicolumn{2}{|c|}{ CSA-DEA results } & \multicolumn{2}{|c|}{ Percen. error } \\
\hline & rfnf & rsnf & rtnf & rcd & $\mathrm{rcl}$ & rcd & $\mathrm{rcl}$ & $r c d$ & $\mathrm{rcl}$ \\
\hline 1 & 0.9960 & 0.9999 & 0.9987 & 0.25 & 0.25 & 0.2451 & 0.24457 & 1.96 & 2.17 \\
\hline 2 & 0.9965 & 0.9945 & 0.9966 & 0.5 & 0.375 & 0.24505 & 0.36663 & 1.98 & 2.23 \\
\hline 3 & 0.99649 & 0.9999 & 0.99509 & 0.333 & 0.5 & 0.3256 & 0.48875 & 2.2 & 2.25 \\
\hline 4 & 0.99979 & 0.9960 & 0.9996 & 0.333 & 0.3125 & 0.3257 & 0.30596 & 2.17 & 2.09 \\
\hline 5 & 0.99982 & 0.9989 & 0.9997 & 0.1667 & 0.3125 & 0.16273 & 0.3052 & 2.36 & 2.33 \\
\hline
\end{tabular}

Table 2. Comparison of the results of CSA, DEA with the hybridised CSA-DEA method

\begin{tabular}{|c|c|c|c|c|c|c|c|c|c|c|c|}
\hline \multirow{2}{*}{ No. } & \multicolumn{4}{|c|}{ FEA results } & \multicolumn{2}{c|}{ CSA results } & \multicolumn{2}{c|}{ DEA results } & \multicolumn{2}{c|}{ CSA-DEA results } \\
\cline { 2 - 13 } & rfnf & rsnf & rtnf & rcd & rcl & rcd & rcl & rcd & $r c l$ & $r c d$ & $r c l$ \\
\hline \hline 1 & 0.9600 & 0.9598 & 0.9590 & 0.24 & 0.3225 & 0.2328 & 0.31266 & 0.2311 & 0.3105 & 0.2353 & 0.3197 \\
\hline 2 & 0.9692 & 0.9690 & 0.9685 & 0.2333 & 0.4375 & 0.2260 & 0.4246 & 0.2243 & 0.4209 & 0.2285 & 0.4286 \\
\hline 3 & 0.9757 & 0.9752 & 0.9748 & 0.225 & 0.45 & 0.2178 & 0.4357 & 0.2160 & 0.43236 & 0.2201 & 0.4405 \\
\hline 4 & 0.9839 & 0.9836 & 0.9832 & 0.2167 & 0.5 & 0.2100 & 0.4839 & 0.2078 & 0.47955 & 0.2121 & 0.49035 \\
\hline 5 & 0.9907 & 0.9894 & 0.9900 & 0.2 & 0.5625 & 0.19344 & 0.5448 & 0.1917 & 0.5390 & 0.19544 & 0.55074 \\
\hline
\end{tabular}

The percentage error between the FEA result and the proposed technique is calculated using the following formula

$\frac{\text { FEA result }- \text { result from the proposed technique }}{\text { FEA result }} \times 100$

The percentage error between the experimental result and the proposed technique is calculated using the following formula

Exp. result - result from the proposed technique

$$
\text { Exp. result }
$$

The convergence behaviour of the proposed method can be observed in Table 1, in which CSA-DEA results are compared with the results of FEM. The errors are found to be within $3 \%$. The error analysis has also been done for Table 2. The errors in DEA are found to be within $4 \%$, and for CSA, the errors are found to be within $3.15 \%$. It can be observed that the percentage of error remarkably drops down to $2 \%$ for the CSA-DEA method. This occurs due to the employment 
Table 3. Results of experimental analysis

\begin{tabular}{|c|c|c|c|c|c|c|c|c|c|}
\hline \multirow{2}{*}{ No. } & \multicolumn{5}{|c|}{ Experimental results } & \multicolumn{2}{|c|}{ CSA-DEA results } & \multicolumn{2}{|c|}{ Percentage error } \\
\hline & rsnf & rsnf & $r \operatorname{tnf}$ & rcd & $\mathrm{rcl}$ & rcd & $\mathrm{rcl}$ & rcd & $\mathrm{rcl}$ \\
\hline 1 & 0.9211 & 0.9219 & 0.9218 & 0.4 & 0.25 & 0.3858 & 0.24075 & 3.55 & 3.7 \\
\hline 2 & 0.9463 & 0.9460 & 0.9458 & 0.367 & 0.3125 & 0.3537 & 0.30115 & 3.62 & 3.632 \\
\hline 3 & 0.9552 & 0.9551 & 0.9549 & 0.2833 & 0.375 & 0.2733 & 0.36206 & 3.53 & 3.45 \\
\hline 4 & 0.9621 & 0.9618 & 0.9616 & 0.25 & 0.4375 & 0.24075 & 0.42056 & 3.7 & 3.872 \\
\hline 5 & 0.9705 & 0.9703 & 0.9700 & 0.2166 & 0.5 & 0.20869 & 0.4812 & 3.652 & 3.76 \\
\hline
\end{tabular}

of crossover and mutation operations from DEA, which results in production of antibodies with potentially good fitness values. The randomisation operation from DEA on the poor and insignificant antibodies improves the fitness values of the antibodies in the next generation, and it helps the algorithm to escape from being trapped in a local solution. It can also be observed during the running of the algorithm that it performs with a good convergence speed without getting trapped in the local solutions. For further justification, the results are also compared with the results of the experimental analysis, as FEA is an approximate method. The results of the experimental analysis are given in Table 3. The error is found to be within $4 \%$. The input variables with the respective outputs are first collected from various methods, and then a set of input variables are treated in the proposed method to get the required outputs. So this is a type of an inverse method which can be used as an inverse engineering problem for fault diagnosis and condition monitoring. The comparison of the results obtained from different techniques are in close agreement with each other having the minimal percentage of errors which shows satisfactory operation of the proposed hybrid algorithm.

\section{Conclusions}

Damage detection using non-destructive techniques has drawn major attention over the last few years. In the current work, a novel hybridisation approach has been proposed using CSA and DEA for fault detection in structural elements. The underlying principles of the standalone techniques are used to create the hybrid model. Here, the first three relative natural frequencies are used as the inputs to the model, and relative values of crack depth and crack location are obtained as the output. The results obtained from the CSA-DEA hybrid model are compared with those from standalone CSA and DEA techniques, and a significant performance improvement has been observed. Finally, the results from the hybrid model are also compared with the results of FEM analysis and experimental analysis, and a close agreement has been observed with the minimal percentage of errors.

\section{References}

1. Abdullah A., Deris S., Anwar S., 2011, Hybrid evolutionary clonal selection for parameter estimation of biological model, International Journal of Computer Applications in Engineering Sciences, 1, 3, 313-319

2. Brest J., MaUČEC M.S., 2011, Self-adaptive differential evolution algorithm using population size reduction and three strategies, Soft Computing, 15, 11, 2157-2174

3. CAddemi S., Morassi A., 2013, Multi-cracked Euler-Bernoulli beams: mathematical modeling and exact solutions, International Journal of Solids and Structures, 50, 6, 944-956

4. Campelo F., Guimarães F.G., Igarashi H., Ramírez J.A., 2005, A clonal selection algorithm for optimization in electromagnetics, IEEE Transactions on Magnetics, 41, 5, 1736-1739 
5. De Castro L.N., von Zuben F.J., 2000, The clonal selection algorithm with engineering applications, Proceedings of GECCO, 36-39

6. Garain U., Chakraborty M.P., Dasgupta D., 2006, Recognition of handwritten indic script using clonal selection algorithm, International Conference on Artificial Immune Systems, 256-266

7. Gong T., 2012, High-precision immune computation for secure face recognition, International Journal of Security and Its Applications (IJSIA), 6, 2, 293-298

8. Gong T., Li L., Guo C., Gong X., 2012, Novel clonal selection algorithm improving selection operator, International Journal of Multimedia and Ubiquitous Engineering, 7, 2, 323-328

9. Guo Z.G., Sun Z., 2011, Multiple cracked beam modeling and damage detection using frequency response function, Structural Longevity, 5, 2, 97-106

10. Ling C.-X., Zhang H.-Q., Lin H., 2009, The modified clonal selection algorithm applied to the remote sensing image information extracting, WRI Global Congress on Intelligent Systems, 2, 94-102

11. Manoach E., Warminska A., Warminski J., 2016, Dynamics of beams under coupled thermomechanical loading, Applied Mechanics and Materials, 849, 57-64

12. Manoach E., Warminski J., Kloda L., Teter A., 2017, Numerical and experimental studies on vibration based methods for detection of damage in composite beams, Composite Structures, 170, 26-39

13. PAWAR R.S., SAWANT S.H., 2014, An overview of vibration analysis of cracked cantilever beam with non-linear parameters and harmonic excitations, International Journal of Innovative Technology and Exploring Engineering, 8, 3, 53-55

14. Pölöskei T., Szekrényes, A., 2017, Quasi-periodic excitation in a delaminated composite beam, Composite Structures, 159, 677-688

15. Pölöskei T., Szekrényes A., 2018, Dynamic stability of a structurally damped delaminated beam using higher order theory, Mathematical Problems in Engineering, DOI: 10.1155/2018/2674813

16. Qin A.K., Suganthan P.N., 2005, Self-adaptive differential evolution algorithm for numerical optimization, The 2005 IEEE Congress on Evolutionary Computation, 2, 1785-1791

17. Rahimi S., Soltani M., 2015, A stochastic simulation algorithm for evaluation of seismic pounding response of adjacent structures, Proceedings of the Institution of Mechanical Engineers, Part O: Journal of Risk and Reliability, 229, 6, 517-529

18. Sinha J.K., Friswell M.I., Edwards S., 2002, Simplified models for the location of cracks in beam structures using measured vibration data, Journal of Sound and Vibration, 251, 1, 13-38

19. Tada H., Paris P.C., Irwin G.R., 1973, The Stress Analysis of Cracks. Handbook, Del Research Corporation

20. TAng Y., 2012, Parameter estimation of Wiener model using differential evolution algorithm, International Journal of Circuits, Systems and Signal Processing, 6, 5, 315-323

21. Timmis J., Hone A., Stibor T., Clark E., 2008, Theoretical advances in artificial immune systems, Theoretical Computer Science, 403, 1, 11-32

22. Xie J., Hu Y., Zhu H., Wang Y., 2013, Surname inherited algorithm research based on artificial immune system, Indonesian Journal of Electrical Engineering and Computer Science, 11, 6, 3194-3199

23. Xu J., Sun K., Xu L., 2015, Data mining-based intelligent fault diagnostics for integrated system health management to avionics, Proceedings of the Institution of Mechanical Engineers, Part O: Journal of Risk and Reliability, 229, 1, 3-15 